\title{
Усиление терагерцовых электромагнитных волн в структуре с двумя слоями графена при протекании постоянного электрического тока: гидродинамическое приближение
}

\author{
(С) И.М. Моисеенко ${ }^{1}$, В.В Попов ${ }^{1}$, Д.В. Фатеев ${ }^{1,2}$ \\ ${ }^{1}$ Саратовский фолиал Института радиотехники и электроники им. В.А. Котельникова Российской академии наук, \\ 410019 Саратов, Россия \\ ${ }^{2}$ Саратовский государственный университет им. Н.Г. Чернышевского, \\ 410012 Саратов, Россия \\ E-mail: MoiseenkolM@yandex.ru
}

Поступила в Редакцию 9 апреля 2021 г.

В окончательной редакции 19 апреля 2021 г.

Принята к публикации 19 апреля 2021 г.

\begin{abstract}
Теоретически исследовано усиление терагерцового электромагнитного излучения в структуре с двумя слоями графена, по которым протекает постоянный электрический ток. Исследована гидродинамическая проводимость графена. Показано, что действительная часть проводимости графена может стать отрицательной в терагерцовом диапазоне частот при скоростях дрейфа носителей заряда в графене, меньших, чем фазовая скорость электромагнитной волны. Для малых волновых векторов падающей терагерцовой электромагнитной волны пространственная дисперсия вносит незначительный вклад в гидродинамическую проводимость графена, из-за чего эффективность усиления волны не зависит он направления токов в каждом из слоев графена. Показано, что графен при протекании постоянного электрического тока может быть использован для создания ТГц-усилителей, работающих при комнатной температуре.
\end{abstract}

Ключевые слова: графен, усиление терагерцового излучения, графен с постоянным током.

DOI: $10.21883 /$ FTP.2021.08.51130.06

\section{1. Введение}

Актуальным направлением по созданию приборов терагерцового (ТГц) диапазона является графеновая фотоника [1]. Графен представляет собой двумерный кристалл, состоящий из атомов углерода, толщиной в один атом, обладающий уникальными свойствами [2-5]. Детекторы и модуляторы ТГц-излучения на основе графена исследуются теоретически [6] и экспериментально [7,8]. Графен с инверсной населенностью носителей заряда может выступать в качестве активной среды для усилителей и генераторов ТГц-излучения [9-11]. Инверсная населенность носителей заряда возникает в графене за счет оптической, инжекционной или диффузионной накачки [11-13]. Благодаря линейному энергетическому спектру графена в результате излучательной рекомбинации электронов и дырок в нем, излучение происходит в ТГц-диапазоне частот. В настоящей работе предлагается отойти от использования механизма достижения усиления в графене за счет создания в нем инверсной населенности ввиду технических и фундаментальных трудностей при создании инверсии в графене, и исследовать альтернативные механизмы усиления ТГц-излучения в графене. В качестве усиливающей среды для ТГц-излучения перспективно исследовать графен с дрейфом носителей заряда, в том числе, благодаря его плазмонным свойствам [14-16]. Усиление в графене с дрейфом носителей заряда может быть достигнуто за счет эффекта Вавилова-Черенкова $[17,18]$. Для этого фазовая скорость электромагнитной волны должна быть меньше скорости дрейфа носителей заряда в графене. Плазмонное усиление в ИК-диапазоне в двухслойной графеновой структуре с дрейфом носителей заряда в одном из слоев графена за счет отрицательного затухания Ландау теоретически исследовано в работах $[19,20]$. В настоящей работе теоретически исследовано усиление ТГц-излучения в структуре с двумя слоями графена, по которым протекает постоянный электрический ток (рассмотрены со- и противоположно направленные токи в разных слоях графена, см. рис. 1).

\section{2. Постановка задачи и метод решения}

Исследуемая структура состоит из двух слоев графена, отделенных слоем диэлектрика толщиной $d$ и

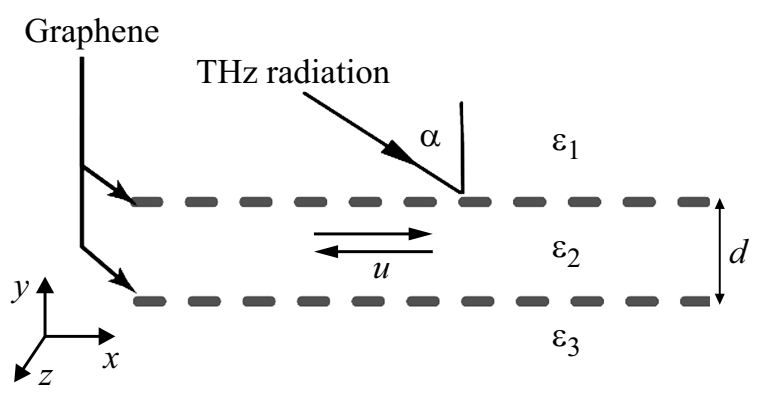

Рис. 1. Схематическое изображение исследуемой структуры. 
окруженных двумя полубесконечными диэлектриками. Применение гидродинамического (ГД) подхода для графена является обоснованным ввиду сильного электронэлектронного взаимодействия в графене на частотах $<5$ ТГц [21]. Электромагнитная ТГц-волна с ТМ-поляризацией (отличные от нуля компоненты электрического и магнитного полей $E_{x}, E_{y}$ и $H_{z}$ соответственно) падает на структуру под произвольным углом (рис. 1). Пространственно-временна́я зависимость электрических и магнитных полей записывается в виде

$$
\begin{aligned}
Y_{x j, y j, z j}= & {\left[Y_{x j, y j, z j}^{+} \exp \left(i k_{y j} y\right)+Y_{x j, y j, z j}^{-} \exp \left(-i k_{y j} y\right)\right] } \\
& \times \exp \left(-i \omega t+i k_{x} x\right)
\end{aligned}
$$

для $j=1,2$ и

$$
Y_{x j, y j, z j}=Y_{x j, y j, z j}^{+} \exp \left(-i \omega t+i k_{x} x+i k_{y j} y\right)
$$

для $j=3$, где $Y_{x j, y j, z j}^{+}, Y_{x j, y j, z j}^{-}-$амплитуды электрического или магнитного полей ТГц-волны и $j-$ номер среды. Тангенциальная составляющая волнового вектора падающей ТГц-волны записывается как $k_{x}=\omega \sqrt{\varepsilon_{1}} \sin \alpha / c$, где $\omega$ и $\alpha-$ соответственно круговая частота и угол падения ТГц-волны, $\varepsilon_{1}$ - диэлектрическая проницаемость среды над графеном, $c$ - скорость света.

Терагерцевый отклик графена для частот ниже частоты электрон-электронных столкновений в графене описывается формулой проводимости, полученной с использованием ГД приближения:

$$
\begin{aligned}
& \sigma\left(k_{x}, \omega\right)= \\
& -\frac{i \varepsilon_{\mathrm{F}} e^{2} \omega\left(2(1-\beta)\left(\omega-k_{x} u\right)-3 i \gamma \beta\right)}{\pi \hbar^{2} \sqrt{1-\beta}\left(k_{x}^{2} V_{\mathrm{F}}^{2}\left(k_{x} u \chi_{1}+\chi_{3}\right)+\omega^{2}(\gamma / \omega-i) \chi_{2}+k_{x} u \chi_{4}\right)},
\end{aligned}
$$

где

$$
\begin{gathered}
\chi_{1}=(2 \beta-1), \quad \chi_{2}=3 \gamma \beta+i \omega(\beta-2), \\
\chi_{3}=\omega-\beta(3 i \gamma+4 \omega), \\
\chi_{4}=\omega(\beta(2 i \gamma-\omega)+(5 i \gamma+4 \omega)),
\end{gathered}
$$

$e-$ заряд электрона, $\gamma=1 / \tau, \tau-$ время релаксации импульса носителей заряда в графене, $\beta=u^{2} / V_{\mathrm{F}}^{2}$, $u$ - скорость дрейфа носителей заряда в графене, $\varepsilon_{\mathrm{F}}$ и $V_{\mathrm{F}}=10^{6} \mathrm{M} / \mathrm{c}$ - соответственно энергия и скорость Ферми в графене. Проводимость графена в ГД режиме получена путем решения уравнений баланса импульса и энергии носителей заряда, а также уравнения непрерывности [14]. Для решения гидродинамических уравнений они линеаризуются, а скорость носителей заряда, эффективная плотность массы электронной жидкости, плотность заряда и плотность тока разлагаются по степеням амплитуды действующего электрического поля с сохранением только стационарного и линейного членов разложения. Электронный дрейф в графене, а также учет давления в ГД электронной жидкости приводит к появлению пространственной дисперсии проводимости графена.
Потоки энергии падающей, отраженной и прошедшей через структуру ТГц-волн определялись как

$$
\begin{gathered}
S_{y}^{\mathrm{inc}}=-\frac{1}{2} \sqrt{\frac{\varepsilon_{0} \varepsilon_{1}}{\mu_{0}}}\left|E_{x}^{\mathrm{inc}}\right|^{2}, \quad S_{y}^{R}=\frac{1}{2} \sqrt{\frac{\varepsilon_{0} \varepsilon_{1}}{\mu_{0}}}\left|E_{x}^{R}\right|^{2}, \\
S_{y}^{T}=-\frac{1}{2} \sqrt{\frac{\varepsilon_{0} \varepsilon_{3}}{\mu_{0}}}\left|E_{x}^{T}\right|^{2},
\end{gathered}
$$

где $E_{x}^{\mathrm{inc}}, E_{x}^{R}, E_{x}^{T}-$ соответственно тангенциальная компонента электрического поля падающей, отраженной и прошедшей волн. Используя выражения (2), запишем коэффициенты отражения и прохождения как $R=\left|S_{y}^{R}\right| /\left|S_{y}^{\text {inc }}\right|$ и $T=\left|S_{y}^{T}\right| /\left|S_{y}^{\text {inc }}\right|$. Тогда коэффициент поглощения можно записать следующим образом: $A=1-R-T$.

\section{3. Результаты расчетов и их обсуждение}

Расчеты проведены для графеновой структуры со следующими параметрами: $\varepsilon_{1}=\varepsilon_{2}=\varepsilon_{3}=4.5$ - диэлектрические проницаемости диэлектриков между слоями графена и их окружения (соответствует часто используемым материалам в структурах графен-диэлектрик), $d=100$ нм - толщина диэлектрика между слоями графена.

На рис. 2, $a$ и $b$ представлена действительная часть гидродинамической проводимости графена $\operatorname{Re} \sigma\left(k_{x}, \omega\right)$ в зависимости от частоты и волнового числа электромагнитной волны, падающей на графеновую структуру. Действительная часть проводимости графена принимает отрицательные значения в ТГц-диапазоне частот, что свидетельствует о возможности усиления электромагнитного излучения в этом диапазоне. Пунктирной линией на рис. 2, $a$ и $b$ показана фазовая скорость электромагнитной волны, совпадающая со скоростью дрейфа носителей заряда в графене $\left(V_{\mathrm{ph}}=\omega / k_{x}=u=0.75 V_{\mathrm{F}}\right)$. В случае, когда фазовая скорость падающей на структуру волны меньше скорости дрейфа носителей заряда в графене $\left(V_{\mathrm{ph}}<u\right)$, усиление в графене достигается за счет эффекта Вавилова-Черенкова. Таким образом, в области слева от пунктирной линии на рис. $2, a$ и $b$ ТГц-усиление связано именно с этим механизмом. Справа от пунктирной линии условие $\operatorname{Re} \sigma\left(k_{x}, \omega\right)<0$ также выполняется, несмотря на то что в этой области фазовая скорость электромагнитной волны превышает скорость дрейфа носителей заряда в графене. В этом случае усиление достигается благодаря передаче импульса и энергии дрейфующих носителей заряда ТГц-волне, взаимодействующей со структурой. Передача импульса происходит через рассеяние на неоднородностях кристаллической решетки графена. Этот факт подтверждается расширением частотного диапазона усиления в сторону повышения частоты при уменьшении времени релаксации импульса носителей заряда в графене (частота $\gamma$ при этом возрастает), при фиксированной скорости дрейфа носителей 

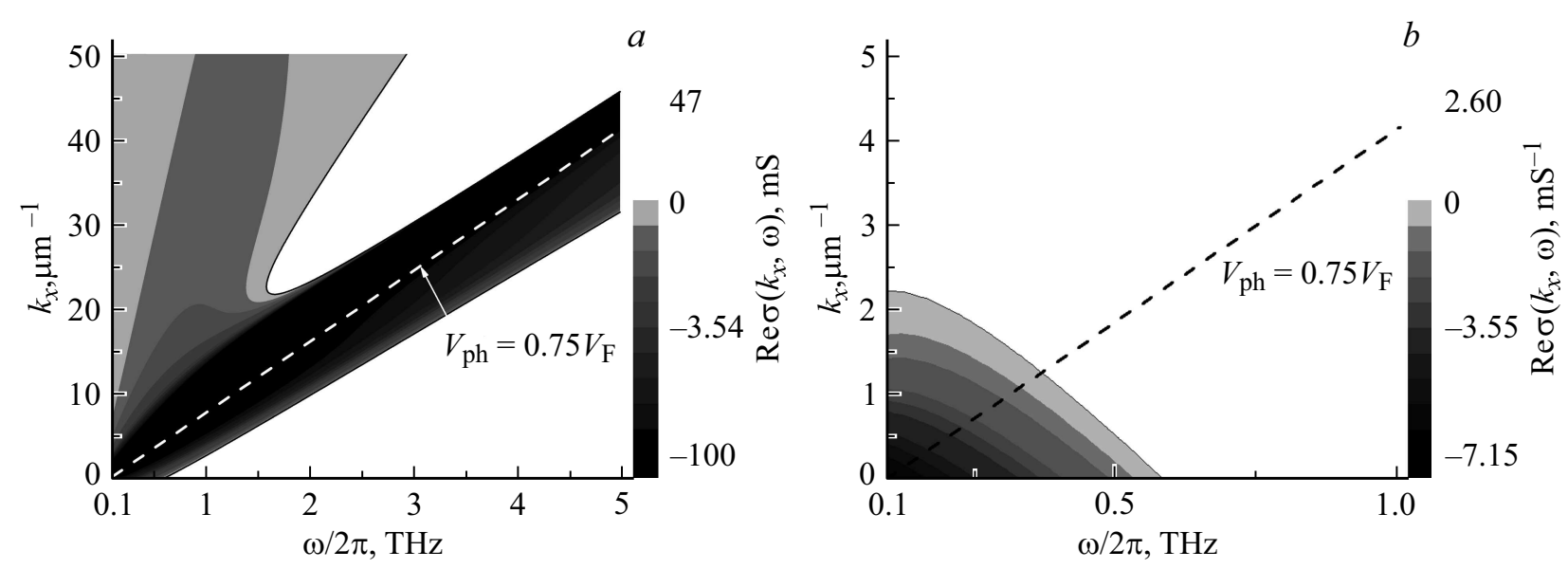

Рис. 2. Действительная часть ГД проводимости графена в зависимости от частоты и волнового числа волны, падающей на графен. Величина времени релаксации импульса носителей заряда и энергии Ферми в графене соответственно $\tau=0.2$ пс и 200 мэВ. Скорость дрейфа носителей заряда в графене показана пунктирной линией и составляет $(a) u=0.75 V_{\mathrm{F}}$ и $(b) u=-0.75 V_{\mathrm{F}}$.

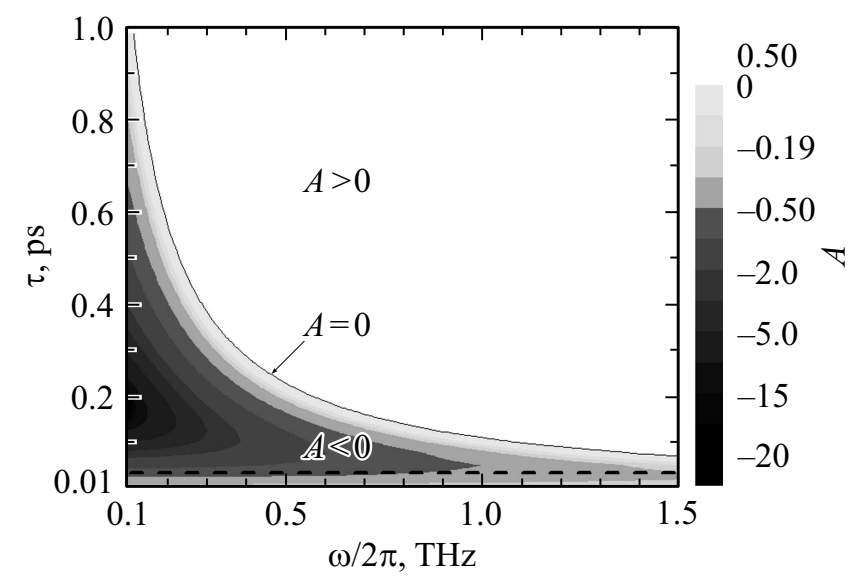

Рис. 3. Спектр поглощения падающей ТГц-волны в двухслойной графеновой структуре в зависимости от времени релаксации импульса носителей заряда в графене. Скорость дрейфа носителей заряда в графене $u=0.75 V_{\mathrm{F}}$, угол падения ТГц-волны $\alpha=35^{\circ}$. Пунктирной линией показана величина $\tau$, соответствующая $\gamma / 2 \pi=1 / 2 \pi \tau=5$ ТГц, определяющая границу применимости гидродинамического приближения.

заряда в графене (рис. 3). Достижение ТГц-усиления в графене за счет постоянного тока выглядит более перспективным по сравнению с усилением в графене с инверсной населенностью, так как для токового усиления нет необходимости иметь высокочистые образцы графена с целью создания долгоживущей инверсной населенности. Если скорость дрейфа направлена противоположно направлению тангенциального волнового числа падающей ТГц-волны (рис. 2,b), то усиление по механизму Вавилова-Черенкова невозможно, однако отрицательная действительная часть проводимости графена говорит о возможном усилении волны.

Если замедление падающей волны мало $\left(k_{x}<0.1 \mathrm{мкM}^{-1}\right), \quad$ то пространственная дисперсия проводимости графена незначительна (величины проводимости с учетом пространственной дисперсии и без нее различаются не более чем на $3 \%$ при $k_{x}<0.1$ мкм $\left.^{-1}\right)$. Для волны с частотой $\omega / 2 \pi=1$ ТГц, падающей под углом $\alpha=35^{\circ}$ из диэлектрика с диэлектрической проницаемостью $\varepsilon_{1}=4.5$ волновое число равно $k_{x}=0.026$ мкм $^{-1}$. Для таких слабо замедленных волн частотная область, где выполняется условие $\operatorname{Re} \sigma\left(k_{x}, \omega\right)<0$, определяется формулой, полученной из уравнения $\operatorname{Re} \sigma\left(k_{x}, \omega\right)=0$ в приближении малых волновых чисел (без учета пространственной дисперсии):

$$
\omega<\gamma \beta \frac{3}{\sqrt{6 \beta+4-7 \beta^{2}}},
$$

для любых скоростей дрейфа $u<V_{\mathrm{F}}$ в графене. Незначительное влияние пространственной дисперсии проводимости графена на спектр усиления слабо замедленных волн в графене также проявляется в отсутствие зависимости частотного диапазона усиления от угла падения ТГц-волны на структуру ( $A=0$ на рис. 4$)$.

На рис. 4 показан спектр поглощения ТГц-волны в зависимости от угла ее падения на графеновую структуру. Усиление в графене соответствует отрицательным значениям коэффициента поглощения $A$ на частотах $\omega / 2 \pi<0.59$ ТГц и происходит за счет отрицательной действительной части проводимости графена. Возрастание коэффициента поглощения по абсолютной величине при уменьшении частоты связано с увеличением по абсолютной величине отрицательных значений действительной части проводимости графена. Важно отметить, что во всей области параметров на рис. 4 фазовая скорость ТГц-волны больше скорости дрейфа носителей заряда в графене.

Эффективность ТГц-усиления возрастает с увеличением скорости дрейфа носителей заряда в графене при фиксированной частоте, падающей на структуру ТГц-волны (рис. 5). При этом частотный диапазон усиления не зависит от направления постоянного тока (вдоль 


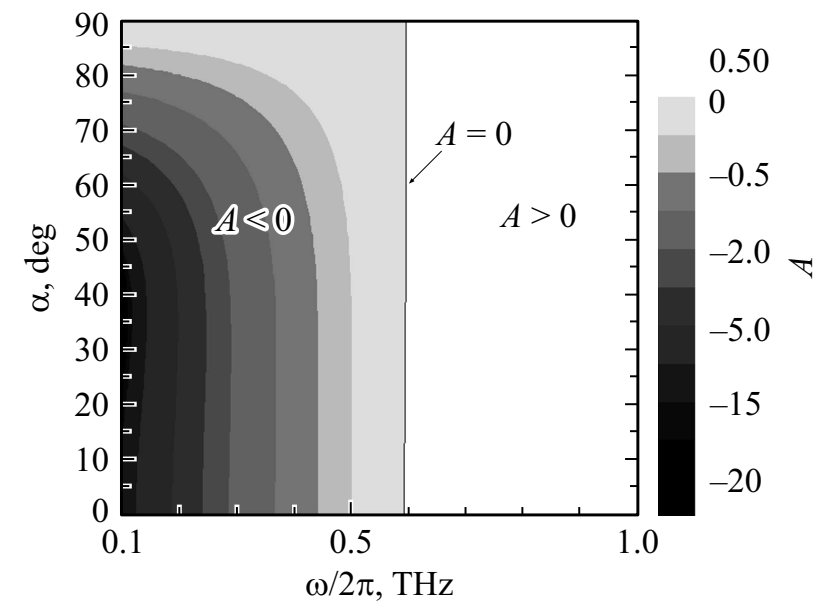

Pис. 4. Спектр поглощения в зависимости от угла падения ТГц-волны на графеновую структуру. Время релаксации импульса носителей заряда и скорость дрейфа носителей заряда в обоих слоях графена соответственно $\tau=0.2$ пс и $u=0.75 V_{\mathrm{F}}$.

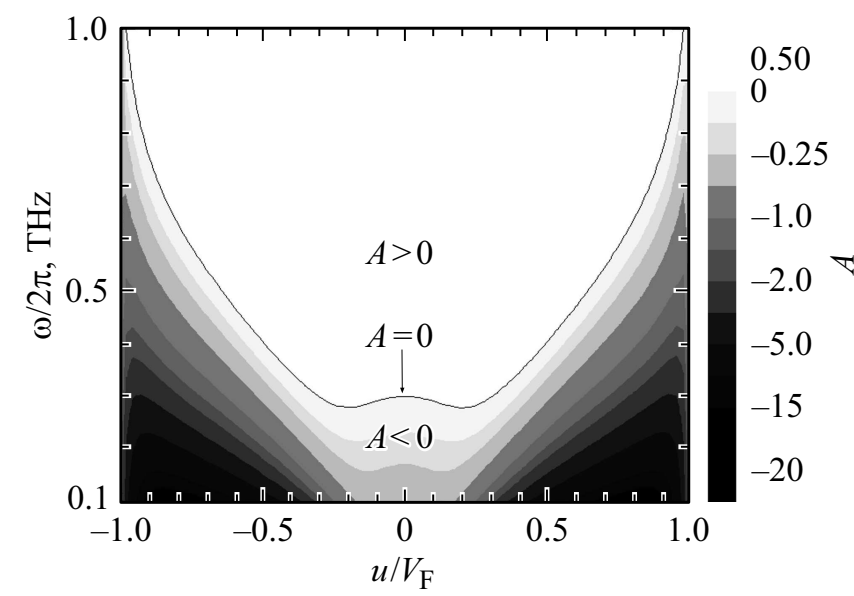

Рис. 5. Спектр поглощения падающей на двухслойную графеновую структуру ТГц-волны в зависимости от скорости дрейфа носителей заряда в одном из слоев графена, нормированной на скорость Ферми в графене, при фиксированном значении скорости дрейфа во втором слое $u=-0.75 V_{\mathrm{F}}$. Время релаксации импульса носителей заряда в обоих слоях графена равно $\tau=0.2$ пс, угол падения волны $\alpha=35^{\circ}$.

или против оси $O x)$ в соответствии с формулой (3), из-за незначительной пространственной дисперсии проводимости графена для слабо замедленных волн. Указанный факт отражается в симметрии спектра поглощения относительно $u=0$ на рис. 5 (слева от $u=0$ токи в обоих слоях графена сонаправлены, справа - противоположно направлены). Усиление при нулевой скорости дрейфа в одном из слоев графена обусловлено отрицательной действительной частью проводимости в другом слое графена, скорость дрейфа в котором зафиксирована на величине $u=-0.75 V_{\mathrm{F}}$. На величину усиления не влияет то, в каком из слоев графена величина постоянного тока зафиксирована, поскольку слои графена расположены достаточно близко друг к другу $\left(l \ll \lambda\right.$, где $\left.\lambda=2 \pi / k_{x}\right)$. Использование двухслойной графеновой структуры приводит к возрастанию эффективности усиления в 2 раза по сравнению с усилением в монослое графена [22] при одинаковых параметрах структуры и характеристиках падающей волны. Таким образом, эффективность усиления ТГц-волны в каждом из слоев графена не уменьшается. Через графен может проходить ток высокой плотности без его разрушения [23]. Для достижения скорости дрейфа носителей заряда в графене $u=0.8 V_{\mathrm{F}}$ необходимо создание электрического поля напряженностью 15 В/мкм. (плотность тока в графене составляет $173 \mathrm{~A} / \mathrm{cm}$ при величине энергии Ферми 200 мэВ). Формула проводимости графена (1) получена в низкотемпературном пределе (энергия Ферми в графене превышает квант тепловой энергии). Все расчеты, приведенные в работе, сделаны для значений энергии Ферми 200 мэВ в обоих слоях графена, что позволяет говорить о возможности ТГц-усиления при комнатных температурах. Стоит отметить, что создание одинаковых значений энергии Ферми не является обязательным условием для достижения ТГц-усиления.

\section{4. Заключение}

Таким образом, в настоящей работе показана возможность усиления электромагнитного ТГц-излучения в структуре с двумя слоями графена, по которым протекает постоянный электрический ток. Исследована ГД проводимость графена, показано, что действительная часть проводимости графена принимает отрицательные значения в ТГц-диапазоне при реально достижимых скоростях дрейфа носителей заряда в графене. В случае слабого замедления падающей на графеновую структуру ТГц-волны пространственная дисперсия вносит незначительный вклад в ГД проводимость графена, из-за чего эффективность ТГц-усиления не зависит он направления токов в каждом из слоев графена. Показано, что величина усиления в двухслойной графеновой структуре в 2 раза превышает величину усиления в монослое графена при одинаковых параметрах структуры и характеристиках падающей ТГц-волны. Показано, что графен при протекании постоянного электрического тока может быть использован для создания ТГц-усилителей, работающих при комнатной температуре.

\section{Финансирование работы}

Работа финансирована стипендией Президента Российской Федерации молодым ученым и аспирантам, осуществляющим перспективные научные исследования и разработки по приоритетным направлениям модернизации российской экономики.

\section{Конфликт интересов}

Авторы заявляют, что у них нет конфликта интересов. 


\section{Список литературы}

[1] J.-H. Son, S. J. Oh, H. Cheon. J. Appl. Phys., 125, 190901 (2019).

[2] A.H. Castro Neto, F. Guinea, N.M.R. Peres, K.S. Novoselov, A.K. Geim. Rev. Mod. Phys., 81, 109 (2009).

[3] S. Das Sarma, S. Adam, E. H. Hwang, E. Rossi. Rev. Mod. Phys., 83, 407 (2011).

[4] A.F. Young, P. Kim. Ann. Rev. Condens. Matter Phys., 2, 101 (2011).

[5] F. Bonaccorso, Z. Sun, T. Hasan, A.C. Ferrari. Nature Photonics, 4, 611 (2010).

[6] O.V. Polischhuk, V. Melnikova V.V. Popov. Appl. Phys. Lett., 109, 131101 (2016).

[7] Q. Guo, R. Yu, C. Li, Sh. Yuan, B. Deng, F.J. Carćia de Abajo, F. Xia. Nature Material, 17, 986 (2018).

[8] P. Olbrich, J. Kamann, M. König. Rev. B, 93, 075422 (2016).

[9] В.Я. Алешкин, А.А. Дубинов, В.И. Рыжий. Письма ЖЭТФ, 89, 70 (2009).

[10] I.M. Moiseenko, V.V. Popov, D.V. Fateev. J. Phys. Commun., 4, 071001 (2020).

[11] O.V. Polischuk, D.V. Fateev, T. Otsuji, V.V. Popov. Appl. Phys. Lett., 111, 081110 (2017).

[12] M.Yu. Morozov, A.R. Davoyan, I.M. Moiseenko, A. Satou, T. Otsuji, V.V. Popov. Appl. Phys. Lett., 106, 061105 (2015).

[13] V. Ryzhii, I. Semenikhin, M. Ryzhii, D. Svintsov, V. Vyurkov, A. Satou, T. Otsuji. J. Appl. Phys., 113, 244505 (2013).

[14] D. Svintsov. Phys. Rev. B, 100, 195428 (2019).

[15] T. Zhao, M. Hu, R. Zhong, S. Gong, Ch. Zhang, Sh. Liu. Appl. Phys. Lett., 110, 231102 (2017).

[16] S. Boubanga-Tombet, W. Knap, D. Yadav, A. Satou, D.B. But, V.V. Popov, I.V. Gorbenko, V. Kachorovskii, T. Otsuji. Phys. Rev. X, 10, 031004 (2020).

[17] H. Fares, M. Almokhtarac. Phys. Lett. A, 383, 1005 (2019).

[18] T.A. Morgado, M.G. Silveirinha. Phys. Rev. Lett., 119, 133901 (2017).

[19] D. Svintsov, V. Ryzhii. Phys. Rev. Lett., 123, 219401 (2019).

[20] T.A. Morgado, M.G. Silveirinha. Phys. Rev. Lett., 123, 219402 (2019).

[21] D. Svintsov. Phys. Rev. B, 97, 121405(R) (2018).

[22] I.M. Moiseenko, V.V. Popov, D.V. Fateev. AIP Conf. Proc., 2300, 020089 (2020)

[23] J. Moser, A. Barreiro, A. Bachtold. Appl. Phys. Lett., 91, 163513 (2007).

Редактор А.Н. Смирнов

\section{Amplification of terahertz electromagnetic waves in a structure with two layers of graphene under direct electric current flow: hydrodynamic approximation}

\author{
I.M. Moiseenko ${ }^{1}$, V.V. Popov ${ }^{1}$, D.V. Fateev ${ }^{1,2}$ \\ ${ }^{1}$ Kotelnikov Institute of Radio Engineering \\ and Electronics, Saratov Branch \\ Russian Academy of Sciences, \\ 410019 Saratov, Russia \\ 2 Saratov State University, \\ 410012 Saratov, Russia
}

\begin{abstract}
The amplification of electromagnetic terahertz radiation in a structure with two layers of graphene with a direct electric current is studied theoretically. The hydrodynamic conductivity of graphene is investigated. It is shown that the real part of the graphene conductivity can be negative in the terahertz frequency range at the drift velocities of charge carriers in graphene that are lower than the phase velocity of the electromagnetic wave. For small wavevectors of a terahertz wave incident on a graphene structure, the spatial dispersion insignificantly contributes to the graphene conductivity. Because of this, the amplification efficiency does not depend on the direction of currents in each of the graphene layers. It is shown that graphene with direct electric current can be used to create $\mathrm{THz}$ amplifiers operating at room temperature.
\end{abstract}

